\title{
Solving Just-in-Time Single Machine Scheduling with Variable Discrete Speed Machine using Hybrid NSGA-II
}

\author{
Bobby Kurniawan ${ }^{a^{*}}$, Ade Irman a, Evi Febianti a, Kulsum a, Lely Herlina a, \\ Muhammad Adha Ilhami a, Yusraini Muharni a, Fellek Getu Tadesse b \\ a Department of Industrial Engineering, Universitas Sultan Ageng Tirtayasa, Benten, Indonesia \\ b School of Mechanical and Industrial Engineering, Ethiopian Institute of Technology-Mekelle, Ethiopia \\ * Corresponding author: b.kurniawan@untirta.ac.id
}

\section{ARTICLE INFO}

\section{Article history}

Received June 1, 2021

Revised August 2, 2021

Accepted August 13, 2021

Available Online August 31, 2021

Keywords

Just-in-time

Single-machine

Variable-speed

Multi-objective

\begin{abstract}
Due to industrialization and population growth, increasing energy demand can lead to energy scarcity because nonrenewable resources are primarily used as energy sources. In addition, carbon dioxide gas, the waste of industrialization, can harm the environment. Therefore, environmentally friendly methods are encouraged in the industrial environment as energy preservation and climate change mitigation. This research discusses just-in-time single machine scheduling that takes into account energy consumption. In this research, energy consumption depends on the machine's speed. The objectives are minimizing the just-in-time (JIT) penalty (the sum of weighted earliness/tardiness) and energy consumption. This research proposed a hybrid NSGA-II with a local search to solve the multi-objective scheduling problem. Thus, solving the JIT singlemachine scheduling problem considers energy consumption to conserve energy and increase production efficiency. Numerical experiments demonstrated that the hybrid NSGA-II with local search is more effective than the standard NSGA-II in solving the problem. Therefore, decision-makers can use the scheduling model to select alternative solutions that consider energy and the environment without sacrificing efficiency.
\end{abstract}

This is an open-access article under the CC-BY-SA license.

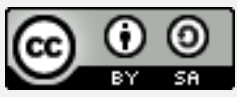

\section{Introduction}

Energy has become a primary need in human life. Energy supports life and drives the economy. However, as the population is growing and the economic development is accelerating, energy consumption increases proportionally. According to the Energy Information Administration (EIA) annual report, energy consumption will increase by $50 \%$ by 2050 . The industrial sector will be the largest energy user with a proportion of around 50\% [1]. Currently, fossil fuels are the primary sources of energy, which are rapidly diminishing due to the increasing energy demand. In addition, the combustion of fossil fuels produces carbon dioxide that is responsible for global warming and climate 
change. Therefore, the growing energy demand can raise two crucial problems that affect human life: energy and the environment.

To reduce the negative impacts of energy consumption, government bodies around the globe have issued energy-efficient policies. For example, renewable energy resources are gradually used to replace non-fossils fuels as an alternative energy source. [2]. Another policy is imposing tariffs according to the demand by incentivizing the private sectors to change their energy consumption patterns or adjusting machine speed $[3,4]$. Being the most significant energy consumer, the industrial sector is required to increase efficiency and reduce consumption. One of the efforts made in efficiency and consumption reduction is by planning and scheduling their operation effectively. As a result, the research has shifted to creating a manufacturing environment that considers energy and sustainability [5-8]. Among the topics in this area, considerable research has been investigating energy-conscious scheduling in a hybrid flow shop [9]. This approach is commonly used in solving energy-conscious scheduling [10-13]. However, because scheduling is known as an NP-hard problem, the use of metaheuristics is redundant [1416]. The idea of making machines idle when the electricity price is high and operating when the electricity price is low has been applied in a single machine scheduling [17]. Stochastic nature in processing and release time has also been considered in the parallel machine scheduling with energy consideration [18]. An energy-conscious single machine scheduling was investigated by Aghelinejad, et al. [19], [20]. This research developed mathematical models to describe the problem and proposed an exact approach for solving them.

As for just-in-time scheduling, Fernandez et al. proposed a constructive heuristic for permutation flow-shop scheduling [21]. Furthermore, a tabu search and variable neighborhood were implemented to solve the hybrid flow shop problems with different time windows [22]. Most scheduling problems assume the non-preemption, i.e., once a job is processed on a machine, it cannot be interrupted. A study investigated this assumption in a single machine scheduling [23]. A mixed-integer programming model for JIT scheduling in an unrelated parallel machine environment was addressed by Xiong et al. [24] solved a flow-shop JIT scheduling in a casting factory. An artificial fish swarm algorithm was proposed to solve the flow-shop JIT scheduling in which the demand was fuzzy [25]. For single-machine JIT scheduling, scheduling and delivery with controllable due dates were solved with a bi-level genetic algorithm [26]. Uncertainty in processing time and due date in a single machine JIT scheduling was investigated by Abedi, et al. [27]. An exact approach was proposed to solve a preemptive single-machine JIT scheduling [28]. A recent survey has been conducted to report the trend in JIT scheduling [29]. Researchers were also interested in single machine JIT scheduling with periodic maintenance [30, 31]. A hybrid approach consists of local search, and a genetic algorithm was proposed to solve the single batch JIT scheduling in a chemical factory[32]. A lower bound approximation for the same problem was proposed by Zhang, et al. [33]. To address problems caused by a machine becoming unavailable, a single machine JIT scheduling was proposed [34].

Inspired by the importance of energy and scheduling to reduce energy consumption, this research investigated single machine scheduling that considers energy usage in a JIT environment. The objective was to minimize the earliness/tardiness penalty while simultaneously reduce energy consumption. Considering that energy consumption is a function of machine speed, a machine that can process jobs faster most likely consumes a considerable amount of energy. On the other hand, a small amount of energy is required to process the job in a normal mode. Thus, the machine speed affects 
the completion time of the job, and in turn, it affects the earliness/tardiness. Given that the scheduling problem has more than one objective function, the hybrid NSGA-II that incorporates local search is proposed to solve the conflicting objectives.

It can be implied that although research in single machine JIT scheduling is ubiquitous [27, 35-37], there has been no previous research that combines the single machine JIT scheduling with energy consumption attributed to the machine's speed. The difference between the research and the previous research on single machine JIT is the objective function. This research aimed to overcome earliness/tardiness penalty and energy consumption with discrete variable speed. Most research in JIT single-machine has focused on the objective of just-in-time (the weighted sum of earliness and tardiness). Furthermore, research has also focused on solving the problem as the singleobjective optimization. Therefore, this research's novelty is addressing the multiobjective JIT single-machine scheduling problem. Most notably, this research considered the energy consumption resulting from the speed of the machine.

Reflecting on the previous discussion, there are several contributions that this research aimed to present. First, this research proposed a mathematical model for the JIT single machine scheduling that considers energy consumption. Secondly, this research employed a hybrid NSGA-II to solve the problem. Lastly, this research confirmed that the proposed method is more efficient in solving the problem than the standard NSGA-II.

In this paper, the discussion is laid out into five parts; Section 1 is the introduction. Section 2 describes the problem, modification of the NSGA-II, and numerical experiments. Section 2 is the results and discussion. The last section concludes this research with further research and research.

\section{Methods}

This section presents the methodology used in this research. First, the problem formulation is described, and the mathematical model is developed (Subsection 2.1). Secondly, the researchers propose the hybrid NSGA-II for solving the problem (Section 2.2). Finally, the researchers designed the numerical experiments to show the effectiveness of the proposed method (Subsection 2.3).

\subsection{Problem Formulation}

A factory has a machine to process jobs or orders from its customers, where $j=$ $1,2, \ldots, N$. The machine's speed can be operated into three modes (indexed by $i$ ): fast, standard, and slow. If the machine is operated using the fast mode, the processing time of a job will be shorter than those in normal or slow mode. However, the machine consumes more energy in fast mode than those in normal or slow mode. The energy consumption of the machine using mode $i$ is denoted by $e_{i}$. Once the machine uses a mode, it cannot be changed until the job is finished.

The processing time of a job is denoted as $p_{i j}$. Every job has a deadline (due date), $d_{j}$. Finally, each job has a start time $S_{j}$ and completion time $C_{j}$. There is a tardiness penalty $\beta$ for completing the job beyond the deadline. On the other hand, the earliness penalty $\alpha$ occurs if the jobs are finished before the deadline. $E_{j}, T_{j}$, and $M$ denote the earliness of a job, tardiness of a job, and the number of speed modes of the machines, respectively.

The scheduling problem assumes the following issues: 
(1) there are no maintenance activities during job processing,

(2) there is no machine breakdown during job processing, and

(3) there is no job preemption, i.e., a job must be processed without disruption.

The previous research indicates that the JIT single machine scheduling problem was investigated initially by Sidney [36]. The research introduced the terminology of earliness and tardiness penalty. Soon after that, many authors developed other models based on this concept.

To develop the mathematical model for JIT single machine scheduling, two binary variables, $x_{i j}$ and $y_{j k}$, and a very big number $V$ are introduced.

$x_{i j}=1$, if job $j$ is processed using speed mode $i, 0$ otherwise.

$y_{j k}=1$, if job $j$ is processed before job $k, 0$ otherwise.

The research designed the following mathematical model.

$$
\operatorname{Min} \sum_{j=1}^{N}\left(\alpha E_{j}+\beta T_{j}\right)+\sum_{i=1}^{M} \sum_{j=1}^{N} e_{i} p_{i j} x_{i j}
$$

subject to

$$
\begin{gathered}
S_{j}+p_{i j} x_{i j} \leq S_{k}+V\left(1-y_{j k}\right), 1 \leq i \leq M, 1 \leq j, k \leq N, j<k \\
S_{k}+p_{i k} x_{i k} \leq S_{j}+V y_{j k}, 1 \leq i \leq M, 1 \leq j, k \leq N, j<k \\
d_{j}-S_{j}-p_{i j} x_{i j} \leq E_{j}, 1 \leq i \leq M, 1 \leq j \leq N \\
S_{j}+p_{i j} x_{i j}-d_{j} \leq T_{j}, 1 \leq i \leq M, 1 \leq j \leq N \\
\sum_{i=1}^{M} x_{i j}=1,1 \leq j \leq N
\end{gathered}
$$

Equation (1) is the objective function that consists of the JIT penalty and energy consumption. Equation (2)-(3) are the disjunctive constraints emphasizing that a machine can process only one job at a time. Equation (4)-(5) calculate the earliness and tardiness of a job. Finally, Equation (6) ensures a job is processed using only one speedmode.

\subsection{Hybrid NSGA-II}

Since the problem concerned in this research is multi-objective optimization, this research proposes a hybrid NSGA-II with a local search to find a non-dominated solution set. The NSGA-II, designed by Deb, et al. [37], is one of the most popular evolutionary algorithm multi-objective methods used for multi-objective optimization problems.

\subsubsection{Encoding}

The encoding uses two vectors of length $N$. The first vector represents the job, while the second vector represents the speed mode used by the machine to process a job. Fig. 1 depicts an individual consisting of 8 jobs and a machine with three-speed modes. Mode 1 indicates the machine is operating at a slow speed, mode 2 indicates standard speed, and mode 3 means that the machine is in fast mode.

\begin{tabular}{|l|l|l|l|l|l|l|l|}
\hline 8 & 3 & 1 & 7 & 6 & 2 & 4 & 5 \\
\hline 1 & 3 & 1 & 2 & 1 & 3 & 2 & 3 \\
\hline
\end{tabular}

Fig. 1. Encoding scheme of an individual 


\subsubsection{Fitness of an individual}

For a single-objective optimization problem, the fitness of an individual can be determined by its objective function. However, a solution of multi-objective optimization is a vector. Thus, it is not easy to compare a solution with others. Therefore, an individual's fitness in multi-objective optimization can be measured by its dominance [37]. Rank and crowding distance is used to determine the dominance of an individual over others. Individuals whom other individuals do not dominate are assigned to rank 1. Furthermore, individuals are given a rank of 2 if they are only dominated by individuals who rank 1. After each individual is categorized by ranking, the individuals in the population are given a crowding distance value. The greater the crowding distance value possessed by an individual, the better the individual is because the individual is located in a less crowded location. Fig. 2 shows the rank and crowding distance.

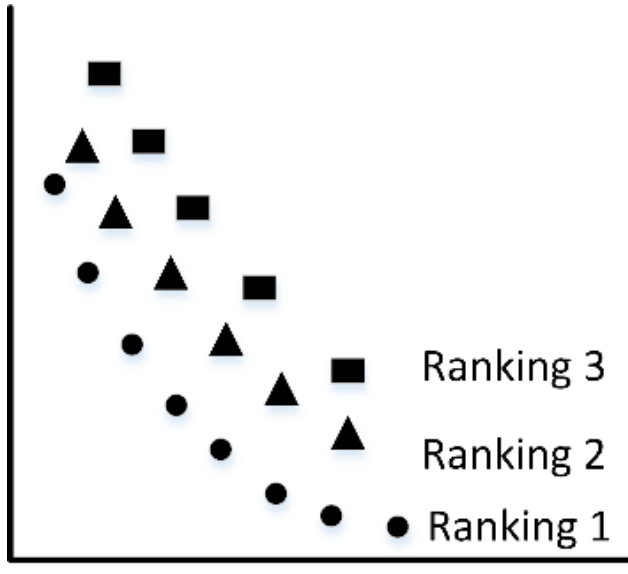

(a)

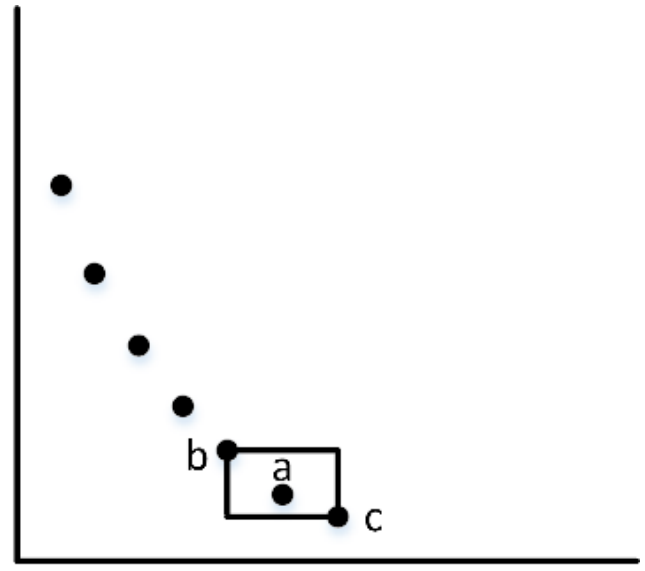

(b)

Fig. 2. Fitness function: (a) rank, and (b) crowding distance

\subsubsection{Selection, crossover, and mutation}

The selection of individuals who will perform crossover is conducted using binary tournament selection. After the binary tournament is performed, the crossover is performed using the single-point crossover method. As for mutations, two types of mutations are carried out. The first mutation is job exchange. The second type of mutation is to change the machine's speed. An example of mutation is illustrated in Fig. 3.

\subsubsection{Local search}

A local search is performed after genetic operations (crossover and mutation) for intensifying the search process. The procedure of the local search is as follows:

1. An individual in rank one is randomly selected as an incumbent.

2. A perturbation is performed on the incumbent. The perturbation is either to change the job exchange or to change the machine's speed.

3. The new individual created from the perturbation is evaluated using dominance criteria. Suppose other individuals do not dominate the new individual. In that case, the new individual is set as the incumbent and stored in the temporary placeholder. 
For the next iteration, the perturbation will be performed on the incumbent. The local search is terminated after it reaches the number of iterations threshold.

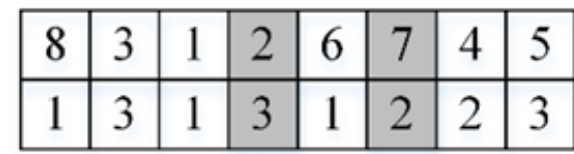

Individual

\begin{tabular}{|l|l|l|l|l|l|l|l|}
\hline 8 & 3 & 1 & 7 & 6 & 2 & 4 & 5 \\
\hline 1 & 3 & 1 & 2 & 1 & 3 & 2 & 3 \\
\hline
\end{tabular}

Mutation

(a)

\begin{tabular}{|l|l|l|l|l|l|l|l|}
\hline 8 & 3 & 1 & 2 & 6 & 7 & 4 & 5 \\
\hline 1 & 3 & 1 & 3 & 1 & 2 & 2 & 3 \\
\hline
\end{tabular}

Individual

\begin{tabular}{|l|l|l|l|l|l|l|l|}
\hline 8 & 3 & 1 & 2 & 6 & 7 & 4 & 5 \\
\hline 1 & 3 & 1 & 2 & 1 & 2 & 2 & 3 \\
\hline
\end{tabular}

Mutation

(b)

Fig. 3. Mutation operator: (a) job exchange, and (b) speed change

\subsubsection{Overall algorithm}

The overall procedure of hybrid NSGA-II with local search is as follows. Let $X, x=$ $1,2, \ldots, X$, be the number of iteration of the hybrid NSGA-II. At initiation $x=0$, the population $Q_{x}$ with the size of popSize is generated randomly. Then, each individual in the population is categorized into rank, and its crowding distance is calculated.

After that, the selection process using binary tournaments is carried out to select individuals for crossover. The crossover produces offspring $Q_{c}$. The following process is mutations on randomly selected individuals. The result of the mutations is denoted as $Q_{m}$. Afterward, a local search is performed on a randomly selected individual. The nondominated individuals obtained from the local search are stored in $Q_{l}$.

The new individuals obtained from crossover, mutation, and local search are combined with the initial population, resulting in a new population for the next generation, $Q_{x+1}=Q_{x}+Q_{c}+Q_{m}+Q_{l}$. The new population $Q_{x+1}$ is sorted based on rank and crowding distance, and as many as popSize from $Q_{x+1}$ is retained for the next evolution. This process is repeated until the number of iterations reaches $X$. The procedure of hybrid NSGA-II is described in Algorithm 1.

\subsection{Numerical Experiment}

\subsubsection{Problem Instance and Parameters}

As many as ten problem instances, i.e., the number of jobs 5, 10, 15, 20, 25, 30, 35, 40, 45, 50, are generated randomly. For each instance, the machine has three modes: slow (mode 1), standard (mode 2), and fast (mode 3). The processing time of each job is a uniform distribution. Random numbers generate the energy consumption of the machine. Soon after the processing time of a job is generated, the due date for a job is calculated using the Equation (7).

$$
d_{j}=H^{*}\left\lfloor\frac{\sum_{i=1}^{M} \sum_{j=1}^{N} p_{i j}}{M N}\right\rfloor
$$


In the Equation, $H$ is a random number between 1.2 and $1.5, M$ is the number of machine speed modes, $N$ is the number of jobs, and $p_{i j}$ is the processing time of job $j$ when processed using mode $i$.

The hybrid NSGA-II uses several parameters with local search. First, the population size popSize is 300 . The crossover and mutation probabilities are 0.75 and 0.01 , and the number of iterations is 300 . Lastly, the NSGA-II algorithm is executed ten times for each instance.

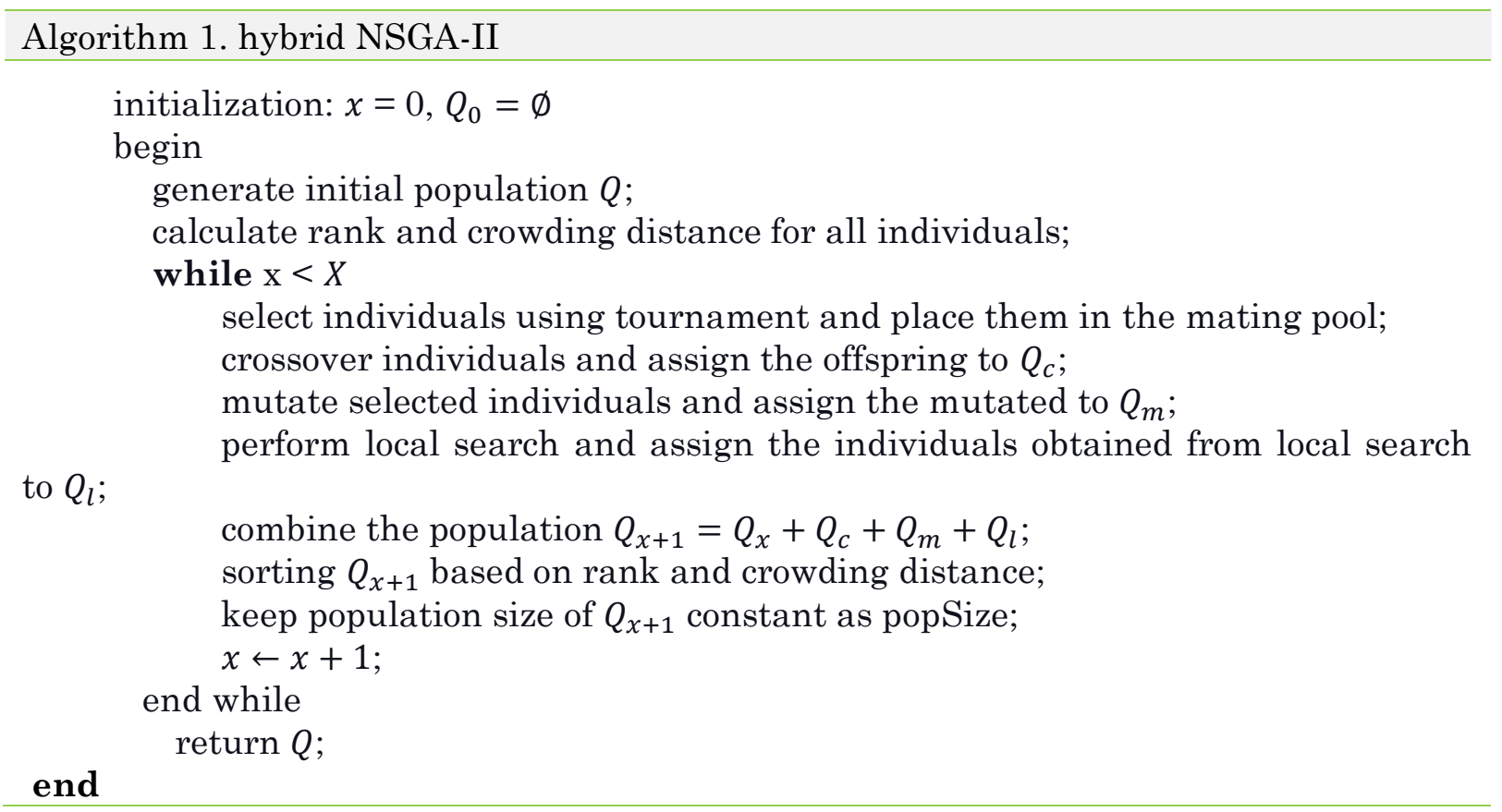

\subsubsection{Performance Metrics}

Two metrics are used to measure the performance of the proposed hybrid NSGA with local search in solving scheduling problems. The first metric is the number of nondominated solutions found by the algorithm in one execution, denoted by $N D$. The larger the $N D$ value, the better the algorithm. The second metric used in this study is the generational distance (GD). GD measures the Euclidian distance from each nondominated solution found by an algorithm $(A)$ with the closest solution from the reference set of solutions (B). GD is calculated using Equation (8).

$$
G D=\sqrt{\frac{\sum_{s=1}^{S} \Delta_{s}}{S}}
$$

To elaborate, $S$ is the number of non-dominated solutions, $s$ is the index of the nondominated solutions, and $\Delta$ is the closest Euclidian distance from the s-th solution to a solution in the reference set. Thus, an algorithm with a small $G D$ value is better than an algorithm that produces a large $G D$. 


\subsubsection{Implementation}

The proposed hybrid NSGA-II with local search is implemented in the JAVA programming language. Numerical experiments were carried out on a hexacore computer with $8 \mathrm{~GB}$ of RAM.

\section{Results and Discussion}

This section discusses the results of the numerical experiments. To evaluate the effectiveness of the hybrid NSGA-II, the research compared the proposed solution with the standard NSGA-II. The encoding, crossover, and mutation of standard NSGA-II were the same as those of hybrid NSGA-II. However, the local search was not embedded in the standard NSGA-II.

\subsection{Analysis of the Effectiveness of the Hybrid NSGA-II with Local Search}

Table 1 displays the results of the numerical experiments. Each instance was executed ten times. For each instance, all non-dominated solutions obtained from 10 runs were combined, and each individual was given a rank. All individuals classified in rank one were stored in the reference set. After that, the GD value for each run was calculated using Equation (8). The $\overline{G D}$ is the average $G D$ of 10 runs for each instance.

Table 1. Numerical results

\begin{tabular}{llllll} 
No & Number of jobs & \multicolumn{2}{c}{ NSGA-II } & \multicolumn{2}{c}{ Hybrid NSGA-II } \\
\hline 1 & 5 & $\overline{\boldsymbol{N D}}$ & $\overline{\boldsymbol{G D}}$ & $\overline{\boldsymbol{N D}}$ & $\overline{\boldsymbol{G D}}$ \\
\hline 2 & 10 & 23 & 0.25 & 38 & 0.13 \\
\hline 3 & 15 & 37 & 1.34 & 43 & 0.67 \\
\hline 4 & 20 & 16 & 2.51 & 22 & 2.71 \\
\hline 5 & 25 & 19 & 3.51 & 18 & 4.79 \\
\hline 6 & 30 & 34 & 10.45 & 46 & 8.63 \\
\hline 7 & 35 & 15 & 25.05 & 17 & 18.73 \\
\hline 8 & 40 & 35 & 35.34 & 49 & 21.43 \\
\hline 9 & 45 & 20 & 45.23 & 15 & 43.85 \\
\hline 10 & 50 & 18 & 45.74 & 21 & 53.01 \\
\hline \multicolumn{2}{l}{ Average } & 15 & 106.2 & 30 & 45.23 \\
\hline
\end{tabular}

From the results in Table 1, it can be concluded that the hybrid NSGA-II with local search was efficient in finding a set of non-dominated solutions. The relatively small average value of $\overline{G D}$ compared with the value found by the standard NSGA-II, which was 19.92 compared with 27.56, confirmed the results. Meanwhile, the ability of the algorithm to find the number of non-dominated solutions, $\overline{N D}$, was quite large, which was 29.9 compared to 23.2. For $\overline{N D}$ metric, the hybrid NSGA-II was better than the standard NSGA-II by $26 \%$. For $\overline{G D}$ metric, the hybrid NSGA-II produced smaller values compared to the standard NSGA-II, at 19.92 to 27.56. Therefore, it can be concluded that the hybrid NSGA-II was more efficient than the standard NSGA-II. The results were influenced by the presence of the local search. 
The GD metric measures how close the solution generated by an algorithm is to a reference set. Thus, the GD metric can be used to describe the accuracy of the resulting solution. The $N D$ metric describes the algorithm's ability to explore multi-objective solutions. The greater the $N D$ value, the better the ability of an algorithm to explore solutions. From Table 1, it can be implied that the accuracy and exploration ability of the hybrid algorithm are satisfactory.

However, the algorithm's limitation is that the greater the number of jobs, the greater the $G D$, due to the scheduling has exponential complexity, i.e., the solution space will increase exponentially as the problem gets bigger.

\subsection{Analysis of the Computation Time}

Fig. 4 shows the average computation time of each problem instance. The greater the number of jobs, the greater the time required. In the case of single-machine scheduling, the computation time is only affected by the number of jobs. It can be seen that the algorithm required less than 150 seconds to find a set of non-dominated solutions for 50 instances.

For each instance, the computation time required by hybrid NSGA-II was longer than that of the standard NSGA-II. The time lag was caused by the time to perform the local search by hybrid NSGA-II. On average, hybrid NSGA-II required approximately $25 \%$ more time than the standard NSGA-II.

\subsection{Managerial Implications}

Decision-makers can have a set of alternative solutions that are tailored to their preferences. Fig. 5 shows several alternative solutions that decision-makers can use in determining the desired solution. In addition, this algorithm can be implemented for solving real-world cases because it can solve a single machine scheduling problem with 50 jobs in less than 150 seconds. Although the hybrid NSGA-II requires more time than the standard NSGA-II, the quality of non-dominated solutions found by the hybrid NSGA-II is beyond NSGA-II. Therefore, this research concluded that a manufacturing company could implement the proposed method that implements a just-in-time production system.

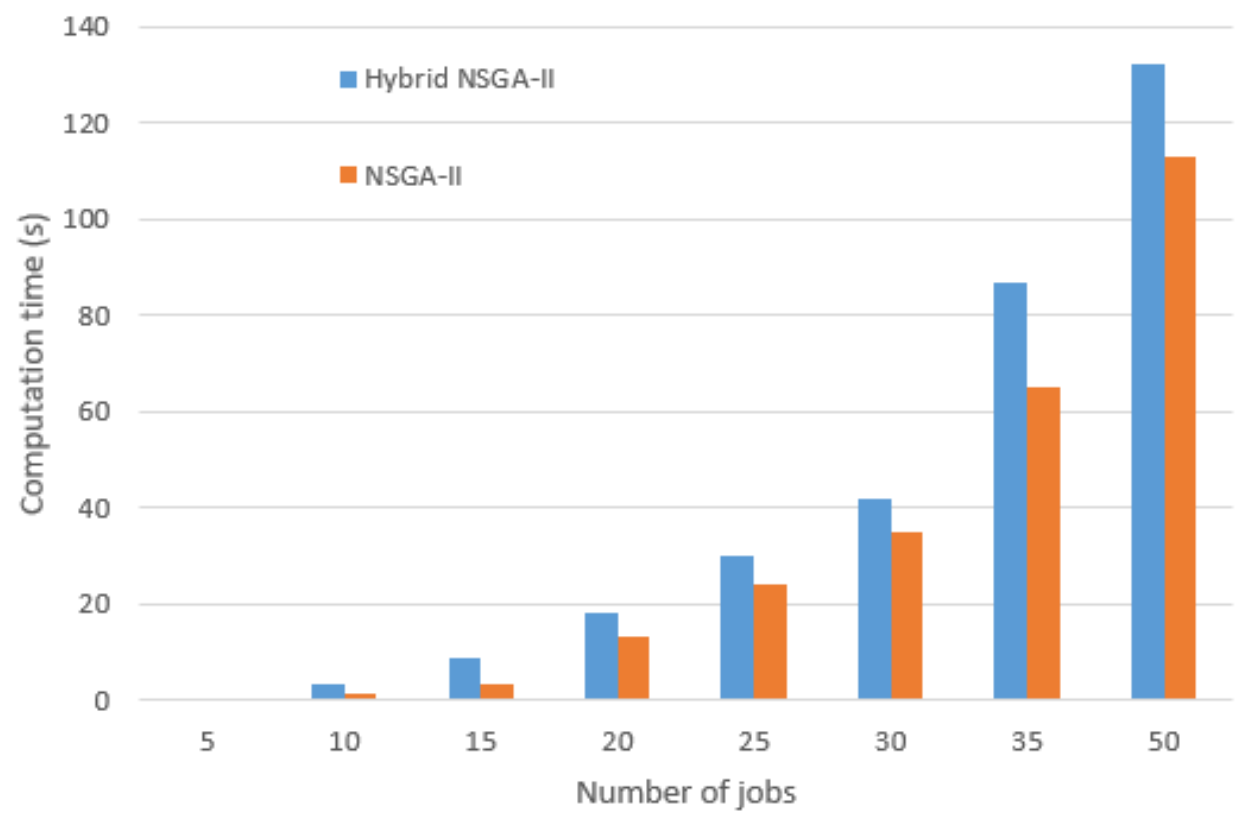

Fig. 4. Computation time 


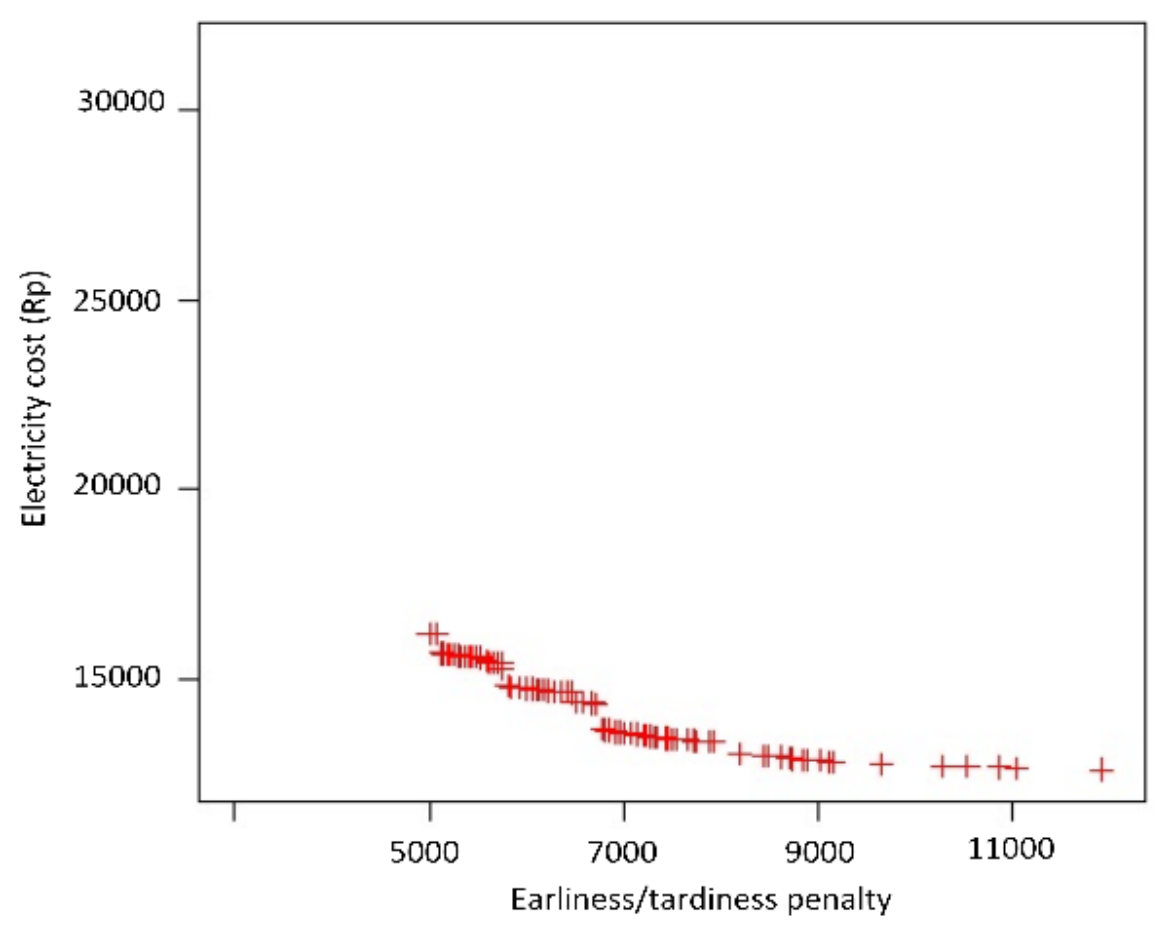

Fig. 5. Distribution of non-dominated solutions of 35 jobs instance obtained by hybrid NSGA-II

\section{Conclusion}

This study addressed multi-objective single machine JIT scheduling with discreet variable speed to minimize earliness/tardiness penalty and energy consumption. The machine can be adjusted into several speed modes. Energy consumption is attributed to the function of machine speed. The hybrid NSGA-II and local search worked effectively to solve this problem. Based on numerical experiments, the hybrid NSGA-II and local search efficiently found a set of non-dominated solutions. The results obtained by the hybrid NSGA-II were compared with those obtained by the standard GA. The scheduling problem can be extended in future research by considering the electricity tariff that depends on the usage time (also known as Time-Of-Use or TOU tariffs). Another possible research area is comparing the hybrid NSGA-II with other methods, such as the multiobjective evolutionary algorithm with decomposition (MOEA/D) or the strength Pareto evolutionary algorithm (SPEA2).

\section{Data Availability}

Raw data are not presented in the paper. If you need the raw data, please contact the author via email.

\section{Declarations}

Author contribution: Bobby Kurniawan conducted Conceptualization, Methodology, Supervision. Ade Irman performed Software running. Evi Febianti carried out Resources, Validation. Kulsum conducted Resources, Visualization. Lely Herlina performed Resources, Validation. Muhammad Adha Ilhami conducted Writing - Review \& Editing. Yusraini Muharni carried out Resources, Validation. Fellek Getu Tadesse performed Writing - Review and Editing. 
Funding statement: This research did not receive any specific grant from funding agencies in the public, commercial, or not-for-profit sectors.

Conflict of interest: The authors declare that they have no conflicts of interest.

Additional information: No additional information is available for this paper.

\section{Acknowledgment}

The authors would like to thank the anonymous reviewers for their helpful suggestions to improve the quality of this paper.

\section{References}

[1] U. E. I. Administration, "International Energy Outlook 2019 with projections to 2050," ed: US EIA Washington, DC, 2019.

[2] M. Götz et al., "Renewable Power-to-Gas: A technological and economic review," Renewable Energy, vol. 85, pp. 1371-1390, 2016. https://doi.org/10.1016/j.renene.2015.07.066.

[3] P. Cappers, C. Goldman, and D. Kathan, "Demand response in U.S. electricity markets: Empirical evidence," Energy, vol. 35, no. 4, pp. 1526-1535, 2010. https://doi.org/10.1016/j.energy.2009.06.029.

[4] K. Fang, N. A. Uhan, F. Zhao, and J. W. Sutherland, "Scheduling on a single machine under time-of-use electricity tariffs," Annals of Operations Research, vol. 238, no. 1, pp. 199-227, 2016. http://doi.org/10.1007/s10479-015-2003-5.

[5] C. Gahm, F. Denz, M. Dirr, and A. Tuma, "Energy-efficient scheduling in manufacturing companies: A review and research framework," European Journal of Operational Research, vol. 248, no. 3, pp. 744-757, 2016. https://doi.org/10.1016/j.ejor.2015.07.017.

[6] M. Akbar and T. Irohara, "Scheduling for sustainable manufacturing: A review," Journal of Cleaner Production, vol. 205, pp. 866-883, 2018. https://doi.org/10.1016/j.jclepro.2018.09.100.

[7] A. N. Sholichah, Y. Yuniaristanto, and I. W. J. J. T. I. Suletra, "Location Routing Problem with Consideration of CO2 Emissions Cost: A Case Study," vol. 21, no. 2, pp. 225-234, 2020. https://doi.org/10.22219/JTIUMM.Vol21.No2.225-234.

[8] A. Dityarini, E. Pujiyanto, and I. W. J. J. T. I. Suletra, "Multi-Objective Optimization Model of Multi-Pass Turning Operations to Minimize Energy, Carbon Emissions, and Production Costs," vol. 21, no. 2, pp. 213-224, 2020. https://doi.org/10.22219/JTIUMM.Vol21.No2.213-224.

[9] A. N. A. A. K. Jabari and A. J. J. T. I. Hasan, "Energy-Aware Scheduling in Hybrid Flow Shop using Firefly Algorithm," vol. 22, no. 1, pp. 18-30, 2021. https://doi.org/10.22219/JTIUMM.Vol22.No1.18-30.

[10] L. Meng, C. Zhang, X. Shao, Y. Ren, and C. Ren, "Mathematical modelling and optimisation of energy-conscious hybrid flow shop scheduling problem with unrelated parallel machines," International Journal of Production Research, vol. 57, no. 4, pp. 1119-1145, 2019. https://doi.org/10.1080/00207543.2018.1501166.

[11] X. Meng, "Lean management in the context of construction supply chains," International Journal of Production Research, vol. 57, no. 11, pp. 3784-3798, 2019. https://doi.org/10.1080/00207543.2019.1566659. 
[12] B. J. J. T. I. Kurniawan, "Mathematical Models of Energy-Conscious Bi-Objective Unrelated Parallel Machine Scheduling," vol. 21, no. 2, pp. 115-125, 2020. https://doi.org/10.22219/JTIUMM.Vol21.No2.115-125.

[13] I. Módos, P. Šůcha, and Z. Hanzálek, "Algorithms for robust production scheduling with energy consumption limits," Computers \& Industrial Engineering, vol. 112, pp. 391-408, 2017. https://doi.org/10.1016/j.cie.2017.08.011.

[14] S. Wang, X. Wang, J. Yu, S. Ma, and M. Liu, "Bi-objective identical parallel machine scheduling to minimize total energy consumption and makespan," Journal of Cleaner Production, vol. 193, pp. 424-440, 2018. https://doi.org/10.1016/j.jclepro.2018.05.056.

[15] D. M. J. J. T. I. Utama, "An effective hybrid sine cosine algorithm to minimize carbon emission on flow-shop scheduling sequence dependent setup," vol. 20, no. 1, pp. 62-72, 2019. http://eprints.umm.ac.id/id/eprint/62683.

[16] S. Chetty and A. O. Adewumi, "A Study on the Enhanced Best Performance Algorithm for the Just-in-Time Scheduling Problem," Discrete Dynamics in Nature and Society, vol. 2015, p. 350308, 2015. http://doi.org/10.1155/2015/350308.

[17] J. Zeng, C. A. Phan, and Y. Matsui, "Supply chain quality management practices and performance: An empirical study," Operations Management Research, vol. 6, no. 1, pp. 19-31, 2013. http://doi.org/10.1007/s12063-012-0074-x.

[18] X. Liu, F. Chu, F. Zheng, C. Chu, and M. Liu, "Parallel machine scheduling with stochastic release times and processing times," International Journal of $\begin{array}{llll}\text { Production Research, } & \text { pp. } & 1-20, & \end{array}$ http://doi.org/10.1080/00207543.2020.1812752.

[19] M. M. Aghelinejad, Y. Ouazene, and A. Yalaoui, "Single-machine scheduling problem with total energy consumption costs minimization $* *$ This research is supported by the Champagne-Ardenne region in France and FEDER (Fonds europen de dveloppement conomique et rgional)," IFAC-PapersOnLine, vol. 52, no. 13, pp. 409-414, 2019. https://doi.org/10.1016/j.ifacol.2019.11.087.

[20] M. Aghelinejad, Y. Ouazene, and A. Yalaoui, "Complexity analysis of energyefficient single machine scheduling problems," Operations Research Perspectives, vol. 6, p. 100105, 2019. https://doi.org/10.1016/j.orp.2019.100105.

[21] A. Ahmadi-Javid, P. Seyedi, and S. S. Syam, "A survey of healthcare facility location," Computers \& Operations Research, vol. 79, pp. 223-263, 2017. https://doi.org/10.1016/j.cor.2016.05.018.

[22] G. A. Idowu, M. O. Adamu, and B. S. Sawyerr, "Hybrid Metaheuristic for Just In Time Scheduling in a Flow Shop with Distinct Time Windows," International Journal of Mathematical Sciences Optimization: Theory Applications, vol. 2020, no. 1, pp. 741-756, 2020. http://ijmso.unilag.edu.ng/article/view/1038.

[23] Y. Hendel, N. Runge, and F. Sourd, "The one-machine just-in-time scheduling problem with preemption," Discrete Optimization, vol. 6, no. 1, pp. 10-22, 2009. https://doi.org/10.1016/j.disopt.2008.08.001.

[24] B. Ogun, Ç. J. J. o. I. E. Alabas-Uslu, and Management, "Mathematical models for a batch scheduling problem to minimize earliness and tardiness," vol. 11, no. 3, pp. 390-405, 2018. http://doi.org/10.3926/jiem.2541.

[25] F. Xiong, M. Chu, Z. Li, Y. Du, and L. Wang, "Just-in-time scheduling for a distributed concrete precast flow shop system," Computers \& Operations Research, vol. 129, p. 105204, 2021. https://doi.org/10.1016/j.cor.2020.105204.

[26] E. B. Tirkolaee, A. Goli, and G. W. Weber, "Fuzzy Mathematical Programming and Self-Adaptive Artificial Fish Swarm Algorithm for Just-in-Time Energy- 
Aware Flow Shop Scheduling Problem With Outsourcing Option," IEEE Transactions on Fuzzy Systems, vol. 28, no. 11, pp. 2772-2783, 2020. http://doi.org/10.1109/TFUZZ.2020.2998174.

[27] M. Abedi, H. J. I. J. o. S. Seidgar, and O. Management, "A new bi-level metaheuristic approach for a single machine JIT-scheduling in the batch delivery system with controllable due dates," vol. 23, no. 2, pp. 135-152, 2016. https://doi.org/10.1504/IJSOM.2016.074052.

[28] O. A. Arık, "Single machine earliness/tardiness scheduling problem with grey processing times and the grey common due date," Grey Systems: Theory and Application, vol. 11, no. 1, pp. 95-109, 2021. https://doi.org/10.1108/GS-01-20200010.

[29] N. M. P. Bocken, P. Rana, and S. W. Short, "Value mapping for sustainable business thinking," Journal of Industrial and Production Engineering, vol. 32, no. 1, pp. 67-81, 2015. http://doi.org/10.1080/21681015.2014.1000399.

[30] G. A. Rolim and M. S. Nagano, "Structural properties and algorithms for earliness and tardiness scheduling against common due dates and windows: A review," Computers \& Industrial Engineering, vol. 149, p. 106803, 2020. https://doi.org/10.1016/j.cie.2020.106803.

[31] R. Benmansour, H. Allaoui, A. Artiba, and S. Hanafi, "Minimizing the weighted sum of maximum earliness and maximum tardiness costs on a single machine with periodic preventive maintenance," Computers \& Operations Research, vol. 47, pp. 106-113, 2014. https://doi.org/10.1016/j.cor.2014.02.004.

[32] M. Shahriari, N. Shoja, A. E. Zade, S. Barak, and M. Sharifi, "JIT single machine scheduling problem with periodic preventive maintenance," Journal of Industrial Engineering International, vol. 12, no. 3, pp. 299-310, 2016. http://doi.org/10.1007/s40092-016-0147-9.

[33] H. Zhang, F. Wu, and Z. Yang, "Hybrid approach for a single-batch-processing machine scheduling problem with a just-in-time objective and consideration of non-identical due dates of jobs," Computers \& Operations Research, vol. 128, p. 105194, 2021. https://doi.org/10.1016/j.cor.2020.105194.

[34] T. Keshavarz, "A lower bounding method for earliness and tardiness minimization on a single batch processing machine," (in en), Journal of Industrial and Systems Engineering, vol. 13, no. Issue 2, pp. 9-26, 2020. http://www.jise.ir/article_113037.html.

[35] C. Low, R.-K. Li, and G.-H. Wu, "Minimizing Total Earliness and Tardiness for Common Due Date Single-Machine Scheduling with an Unavailability Interval," Mathematical Problems in Engineering, vol. 2016, p. 6124734, 2016. http://doi.org/10.1155/2016/6124734.

[36] J. B. J. O. R. Sidney, "Optimal single-machine scheduling with earliness and tardiness penalties," vol. 25, no. 1, pp. 62-69, 1977. https://doi.org/10.1287/opre.25.1.62.

[37] K. Deb, A. Pratap, S. Agarwal, and T. Meyarivan, "A fast and elitist multiobjective genetic algorithm: NSGA-II," IEEE Transactions on Evolutionary Computation, vol. 6, no. 2, pp. 182-197, 2002. http://doi.org/10.1109/4235.996017. 